\title{
Comparative Study on Learning Space of Learning Buildings at Universities based on Visibility
}

\author{
Wang Jiahui ${ }^{1, a}$, Wu Fang ${ }^{1, b,{ }^{*} \text { Bao Ziyang }}{ }^{1, c}$ and Bao Lingxue ${ }^{1, d}$ \\ 'Zhejiang University City College, Department of Architecture, \\ Hangzhou City, Zhejiang Province, China \\ a495834536@qq.com, bwuf@zucc.edu.cn, c493761376@qq.com, d691918952@qq.com \\ ${ }^{*}$ Corresponding author
}

Keywords: Learning Space, Visibility, a processing mode for bracing spaces.

\begin{abstract}
Recently, the studies on learning space to explore the operational mechanism and design method are rare. Meanwhile, the analysis methods of atriums and courtyards are not clear. In this study, a new model is proposed to distinguish the void space in the visible analysis which is based on two case studies. Furthermore, the operational mechanism and space feature will be discussed according to the visual characteristics of the spatial structure. Following contents should be emphasized in the process of the learning environment design: 1 . The relationship between the layout of public streamline space and local space; 2. Coupling between the spatial distribution of learning space and the spatial visibility characteristics; 3. Synergy and effective enhancement among different learning spaces.
\end{abstract}

\section{Preface}

\subsection{Introduction}

In the changing age of information, the boundary between teaching space and public space is increasingly blurred. Those traditional design methods solely depending on perceptual experience can no longer meet future requirements. Scientific quantification makes special measurement standards more convincing. The research is based on space syntax theory and related tools (UCL Depthmap).

\subsection{Review of related research and development}

The syntactic analysis of sight in Depthmap, it is the theoretical foundation of this research. The correlation among visibility, accessibility and calculation methods has been used generally (Carranza et al, 2013; Lu, 2010; Lu et al, 2009; Morgareidge et al, 2014)[2][3][4]. Setola et al. (2013) [5] Through the interaction density and accessibility diagram, the relationship between building users is shown. VGA helps to understand cultural differences in architectural design (Cai and Zimring, 2013).

Measurement of visual area index studies the mathematical characteristics of the visual area based on human spatial perception (Stamps, 2005) [7]. Some of these indicators have been positively and statistically significant correlations with spatial perception (Dawes and Ostwald, 2014; Dosen and Ostwald, 2017) [8] [9].

Unfortunately, few studies have focused on the visibility of the learning space to explore its operational mechanism and design methods. Meanwhile, analysis methods of space of atriums and courtyards are not clear, while some even do not participate in analysis process (Ju Hyun Leea and Michael J. Ostwald, 2017). The corresponding analysis method needs further study. 


\section{Research method}

\subsection{Visibility analysis}

In this study, two cases are focused separately including learning building at new flagship campus in Westminster, UK and No. 4 learning building of science and engineering major at urban institute of Zhejiang University (hereinafter referred to the British case and the Chinese case). First and fourth floors are extracted from the two cases under names of UK1, UK2, CN1 and CN2 respectively. (Shown in Table 1)

Table 1. Chinese and English cases background data sheet.

\begin{tabular}{|c|c|c|c|c|}
\hline $\begin{array}{c}\text { National } \\
\text { Building }\end{array}$ & \multicolumn{2}{|c|}{$\begin{array}{c}\text { new flagship campus in } \\
\text { Westminster, UK }\end{array}$} & \multicolumn{2}{c|}{$\begin{array}{c}\text { No. } 4 \text { learning building of science and engineering major at } \\
\text { urban institute of Zhejiang University }\end{array}$} \\
\hline No. & UK1 & UK2 & CN1 & CN2 \\
\hline Project Year & \multicolumn{2}{|c|}{2010} & & 2006 \\
\hline Area[m²] & 4688 & 3776 & 2432 & 3835.5 \\
\hline
\end{tabular}

In the process of human environmental cognition, visibility plays a vital role. In this study, it is significant to propose two concepts: visibility and accessibility. For instance, according to the definition above, the indoor atrium is visually inaccessible. Therefore, it should be considered into the visual analysis, but which can not be used effectively. The outdoor courtyard space is more complex. In this study, two hypothetical research schemes will be proposed to comparison.

Scheme 1: bracings do not be involved in software operation, no data generated

Scheme 2: bracings involve in calculation, but the corresponding data in the range do not be involved in the statistical analysis. In performance, it is expressed as transparency 50\%. (Table 1direct correspondence analysis)

The partition in public space will lead to different effects due to the transparency and height of materials. For example, a transparent glass wall in the information center is considered visible, and a screen above the human view in the display area is considered invisible. (Shown in Fig. 1 - direct correspondence analysis)

\subsection{Visual area analysis}

Four types of special locations could represent four user perspectives: visitors, formal learners, informal learners and shared space experiencers. Atrium space is included in the visual area and special processing is not included while graphic expressing and data processing.

\subsection{Space types of 6 learning areas}

Learning space is classified according to potential learning behavior. The space could be divided into general education areas, specialized education area, gathering area, sharing area and outdoor area. Main activity areas are our concerns.

\section{Results}

\subsection{Visual analysis}

Data analysis is divided into three dimensions: the overall mean, the overall distribution, the spatial distribution and the relationship with learning space. The UK2-1 indicates that the atrium space of UK2 is handled by scheme 1. UK2-2 is designed to adopt scheme 2 (shown in figure 1). CN2-1 is the same as UK2-1. UK2-2 has one interior hall space; CN2-2 has two interior hall spaces (No. 1, No. 2) and two outdoor courtyard spaces (No. 3, No. 4).

3.1.1 Connectivity analysis

3.1.1.1 Overall mean value

In the UK case, the CON mean of UK2-1 was slightly larger than UK 1, while UK2-2 was 80.1\% higher than UK2-1; but the UK2-1 study area was the same as that of UK2-1 and slightly smaller than UK-1. In the Chinese case, CN2-2 is $194.3 \%$ higher than CN-2 and 64.38\% larger than CN-1, while research area of CN2-2 was the same as CN2-1. It can be seen that the determinants of CON mean are 
not the size of space, but the layout of spatial morphology and the visibility relationship between local spaces.

The study area of UK1 is larger than that of CN1, but the spatial shape of UK1 is more like a dumbbell-shaped string layout, while CN1 is more like a plate-shaped linear layout. So, CN1 is higher than UK1 in CON mean attributes and more open; but comparing with UK-2 and CN-2, UK1 and $\mathrm{CN} 1$ are located at the bottom of the more spatial isolation and hierarchy, while UK2-2 and CN2-2 are visually inclusive, as well as more conducive to enriching communication and learning activities.

Table 2. Chinese and English visibility graph analysis tables.

\begin{tabular}{|c|c|c|c|c|c|c|c|c|}
\hline & UK1 & UK2-1 & UK2-2 & comparison & CN1 & CN2-1 & CN2-2 & comparison \\
\hline $\begin{array}{c}\text { Analysis } \\
\left.\text { area [m }{ }^{2}\right]\end{array}$ & 1315 & 1146 & 1146 & & 728 & 1050 & 1050 & \\
\hline CON & 1021.29 & 1039.55 & 1872.64 & 0.801 & 1381.94 & 785.88 & 2313.14 & 1.943 \\
\hline VMD & 2.18 & 2.37 & 1.93 & -0.186 & 1.64 & 2.14 & 1.89 & -0.117 \\
\hline VCC & 0.68 & 0.76 & 0.77 & 0.013 & 0.78 & 0.84 & 0.69 & -0.179 \\
\hline
\end{tabular}

Con: Connectivity, VCC: Visual Clustering Coefficient, VMD: visual mean depth.

\subsubsection{Overall layout}
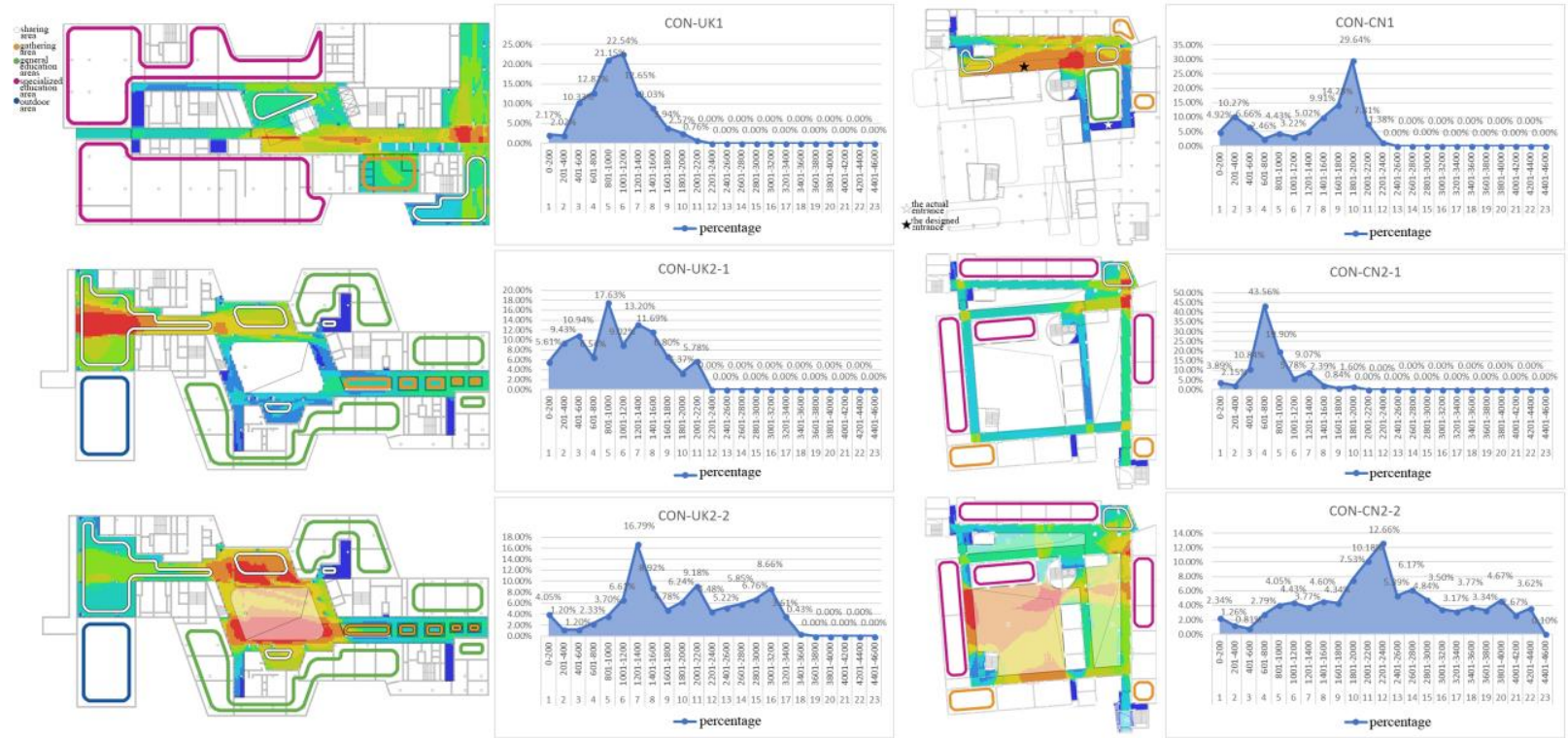

Fig. 1. Comparison of connectivity distribution differences between Chinese and English cases.

In the UK case, the distribution range of UK2-2 (0-3600) is much larger than that of UK1 and UK2-1 (0-2200), and the main range of CON values is 1000-3400, which is closer to the right than UK-1 and UK2-1. It indicates that the overall level of CON in UK2-2 is better than that of UK1 and UK2-1; for UK-2, the proportion of low CON space is significantly reduced compared with UK2-1.

In Chinese case, $43.56 \%$ of CN2-1 fell in the range of $600-800 \mathrm{CON}$ and $74.3 \%$ in the range of 400-1000, indicating changes on range of visual area are small, and the visual experience in flow process of space is similar. The peak in CN2-2 shifts to the right than that in CN1 and CN2-1, indicating that the overall level of $\mathrm{CON}$ has been greatly improved. Therefore, CN2-2 also has more types of visual areas with more hierarchical and exploratory features.

Combing with plan, high CON spaces of UK2-2 and CN2-2 are concentrated around the courtyard. Bracing spaces of CN2-2 (indoor and outdoor) cover more largely than UK2-2. Thus, the processing methods of proportion of areas are important reasons that affect the proportion of high value of CON distribution interval. 


\subsubsection{Spatial distribution and relationship with learning space}

The shared space and aggregated learning space in UK1 are mainly located on one side of the high CON space, having good visual guidance; entrance and exit of the specialized education space are mostly located in the low CON area, having better visual concealment.

The high-CON space of UK2-1 is far away from the atrium area, while the low CON space mainly appears in the end of corridor; the high-CON space of UK2-2 is located around the atrium space, and the spatial distribution of low-CON is similar to UK2-1. Comparing with onsite photos, UK2-2 can reflect the visual experience of the scene better. In UK2-2, four shared learning areas are located in different CON attribute areas, having advantages of meeting needs from different groups of people (public/private, noisy/quiet, fast accessibility); ordinary classrooms are located in different CON attribute areas, which are able to become formal and informal learning group areas with their adjacent shared learning areas. It is convenient to realize transformation of learning patterns; outdoor learning spaces are located on the side of the middle CON attribute area adjacent to the largest shared learning areas, which are available to form synergy effect.

Actual population flow entrance and etiquette exit of $\mathrm{CN} 1$ are separated actually, while actual flow entrance areas are basically the lowest $\mathrm{CON}$, indicating the second entrance of CN1 is weak at visual guidance. The shared space and aggregate learning spaces of this layer are mainly located in the high CON spaces or one side, having good visual guidance. The high-CON space of CN2-1 is located at the intersection of the road in the upper right corner of the plane, the CON of the corridors around the bracing spaces are not very high, while spaces of low CON appear mainly in the end of corridors; high CON spaces of CN2-2 are located in the corridor around the outdoor courtyard, especially the left and lower corridor of Courtyard 3 and the right corridor of Courtyard 4 . The spatial distribution of low $\mathrm{CON}$ is similar to $\mathrm{CN} 2-1$. Comparing with onsite photos, CN2-2 can reflect the actual experience of onsite atrium 1 and 2, while the outdoor compound courtyard is between CN2-1 and $\mathrm{CN} 2-2$, closer to $\mathrm{CN} 2-2$. Based on on $\mathrm{CN} 2-2$, this paper analyzes the distribution of learning space. Two gathered learning areas are located on the side of the lower CON area which are not easy to find; among 4 specialized education spaces, 2 of them are located in the high CON areas, while another 2 are located in middle CON areas. For high CON areas, outdoor courtyard scenes could be seen. It is impossible to not observe the existence of peripheral informal learning facilities, and in fact, there is no such configuration around. Thus, formal and informal learning group areas could not be built effectively, while transformation of learning modes can not be achieved.

\subsubsection{Analysis on visual mean depth}

\subsubsection{Overall mean value}
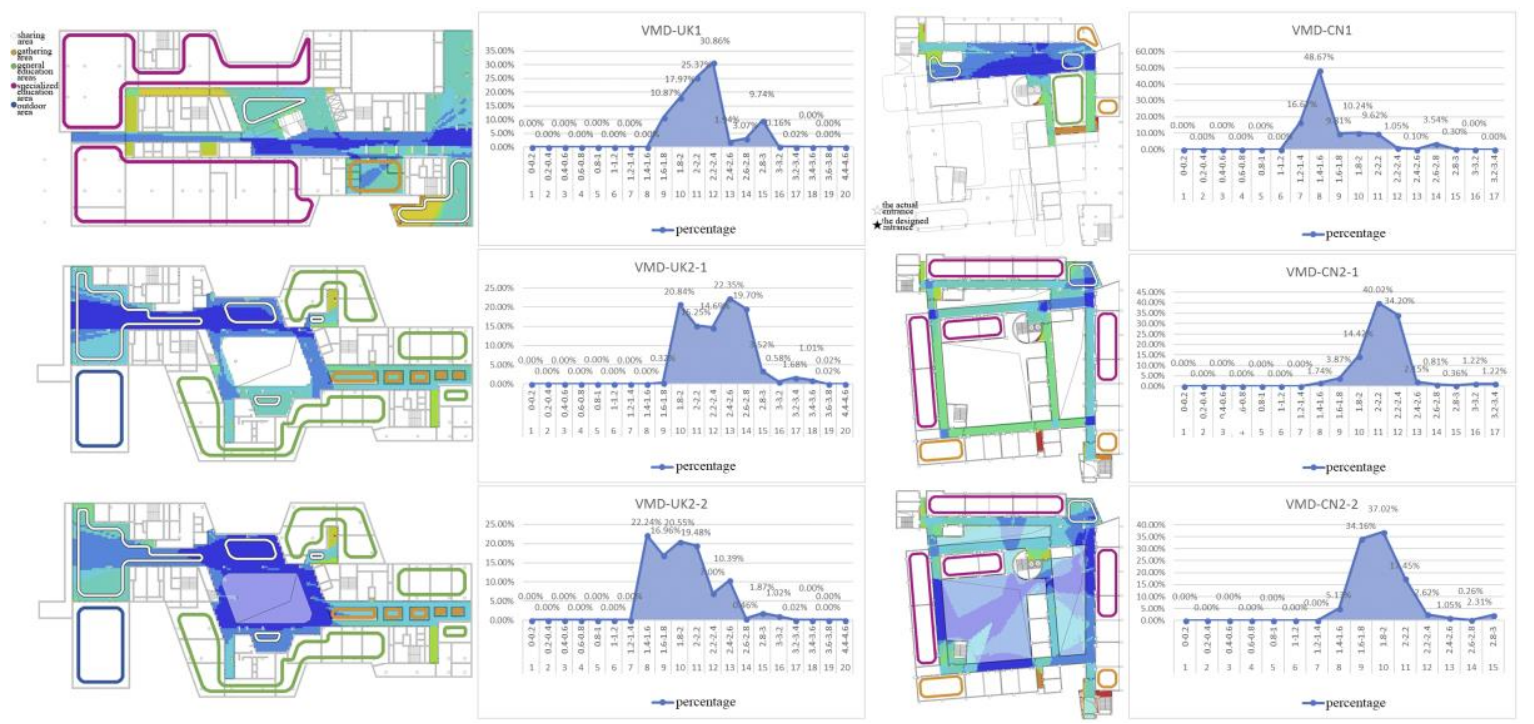

Fig. 2. Comparison of visual average depth distribution differences between Chinese and English cases. In the UK cases, VMD mean values of UK2-1 is slightly larger than UK2-2, and similar to UK1; both have the same proportion and slightly smaller than UK1 (seen as table 2) of Chinese cases, while mean value of CN 2-1 VMD is $11.7 \%, 23.36 \%$ higher than $\mathrm{CN} 1$; CN2-1 research area is larger than 
$\mathrm{CN} 1$, which is the same as CN2-2. It could be seen that important factors affecting the mean value of VMD are the size of the space area, the layout of space morphology and the visibility relationship between local spaces.

\subsubsection{Overall distribution}

In English cases, the distribution shape of UK 1 is multi-peak (see Figure 2), while UK2-1 and UK2-2 are performing in platforms, indicating UK2-1 and UK2-2 having more homogeneous VMD distribution. UK2-2 (1.4-2.6) was left-sided more than UK2-1 (1.8-3) and UK1 (1.6-3) VMD, suggesting that the overall VMD in UK2-2 was smaller than UK1 and UK2-1. It is easier to observe the surrounding learning facilities and environment.

In the Chinese case, $75.15 \%$ of $\mathrm{CN} 1$ fall between 1.2 and 1.8, 74.20\% of CN2-1 fall between 2-2.4 and $71.18 \%$ of CN2-2 fall between 1.8 and 2. It indicates that learning facilities in $\mathrm{CN} 1$ are the easiest to find, while learning facilities and environment of CN2-2 are easier to find than CN2-1. This is consistent with the difference in VMD mean value.

Low VMD spaces of UK2-2 and CN2-2 appear in the vicinity of atriums and courtyards. The two courtyards in CN2-2 interact with each other, and the proportion of the coverage area is greater than UK2-2. Thus, the bracing processing methods and proportions are mean reasons affecting low proportion of VMD distribution intervals.

\subsubsection{Spatial distribution and relationship with learning spaces}

Shared space and aggregated learning spaces of UK1 are located in low VMD spaces which are easy to observe. At the end of the shared learning area in the lower right corner is the coffee lounge with deeper VMD. But, because it is near to shared spaces of VMD, it can be both accessible and private.

Low VMD areas of UK2-1 are mainly located at the left end of the plane and the adjacent atrium corridor. Low VMD spaces of UK2-2 are located around the atrium spaces. Comparing with onsite photos, UK2-2 can reflect the visual experience of the scene better. In UK2-2, the four shared learning areas are basically in low VMD areas, which are easy to find and use.

Low VMD spaces of CN2-1 are located at the intersections of roads in the upper right corner of the plane, while the low VMD spaces of CN2-2 are located in the corridors around the outdoor courtyard. Comparing with onsite photos, CN2-2 could reflect the scene. One shared learning area in CN2-2 is located on the side of low VMD area, which was low in VMD, easy to detect but poor in privacy.

3.1.3 Clustering coefficient analysis

\subsubsection{Overall mean values}
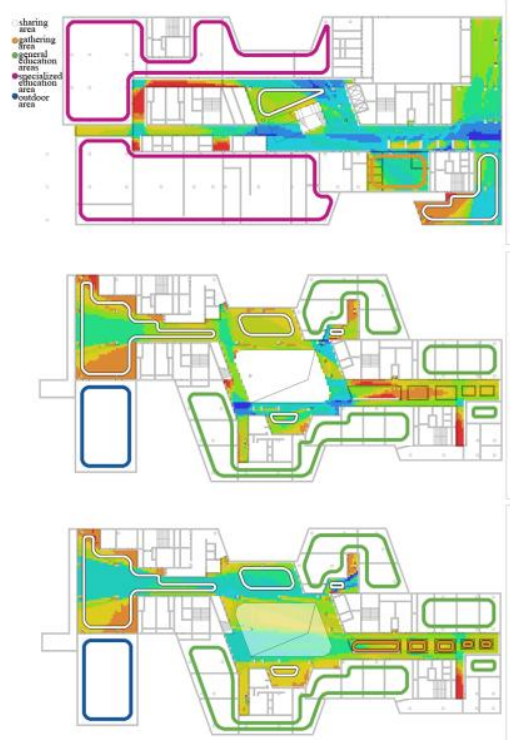

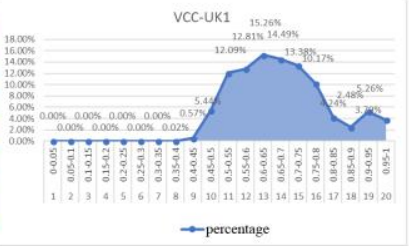

VCC-UK2-1
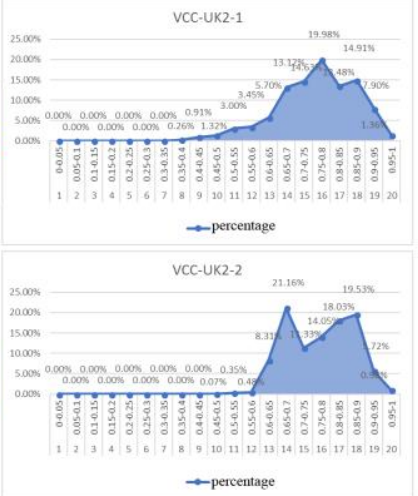
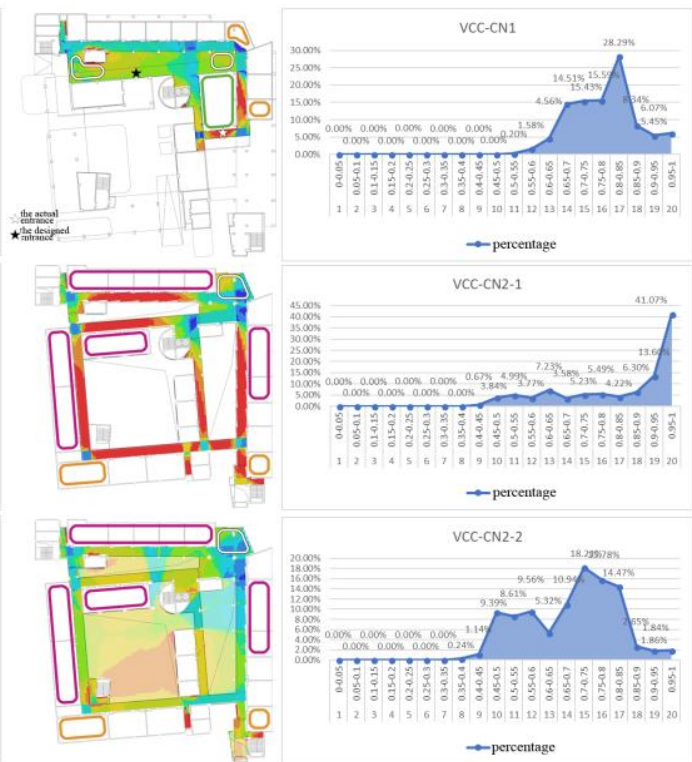

Fig. 3. Comparison of distribution differences on Visual Clustering Coefficient (VCC) from Chinese and English cases.

In the UK cases, VCC mean values of UK2-1 and UK2-2 are similar (seen in figure 3) and larger than UK1; but study area of UK2-1 is slightly smaller than UK1, while study areas of UK2-2 is the same as UK2-1. In Chinese cases, VCC mean value of CN2-1 is the largest, followed by CN1, and CN2-2 is 
the smallest. CN2-2 is $17.9 \%$ smaller than the mean value of $\mathrm{CN} 2-1$; however, the research area of $\mathrm{CN} 2-1$ is the same as CN2-2 and larger than CN1. Therefore, the size of the space is still not the decisive factor affecting the VCC mean value.

\subsubsection{Overall distribution}

In UK cases, comparing with UK2-1, the proportion of low VCC spaces of UK2-2 is decreased significantly and the possibility of visual mutation is less, while the spatial guidance is lower. In Chinese cases, the peak of CN2-1 obviously shifts to the right side comparing with CN1 and CN2, while CN2-1 also has an extreme possibility of visual mutation and special guidance, mainly reflecting on intersections of middle corridors and corridors.

Based on comparison on UK and Chinese cases, value span of the three is the same, but the VCC value span of CN2-1 mainly fall on the range of 0.9 to 1 , which is closer to the right than UK2-2 (0.6-0.95) and CN2-2 (0.45-0.85). This indicates that the highest VCC space in the main space of CN2-2 is the smallest, followed by UK2-2, and CN2-1 is the largest. Most of low VCC spaces of UK2-2 and CN2-2 appear near the bracing spaces. Thus, the visual property of corridor interface is an important factor that affects the proportion of the high value of VCC distribution interval.

\subsubsection{Spatial distribution and relationship with learning space}

For the most important formal learning space analysis, VCC of specialized education space of CN2-1 is very high (0.9-1), while CN2-2 is relatively slow (0.55-0.85), which means front visual line of formal learning space in CN2-1 basically has not changes with worse special guidance based on consideration of plane visibility. This proves that the CN2-2 model is closer to the actual situation. Only one shared learning area of CN2-2 is located on one side of the lower VCC area, and is easy to observe.

\subsection{Visual area analysis}

Four special locations are selected to analyze the visual areas, and four indexes are calculated on the basis of which: visual areas proportions (the average value of the area of buildings that can be seen when an agent is walking in sequence and viewed from a 360-degree view angle), briefly as IA [10], IDM and IO (seen as figure 4).

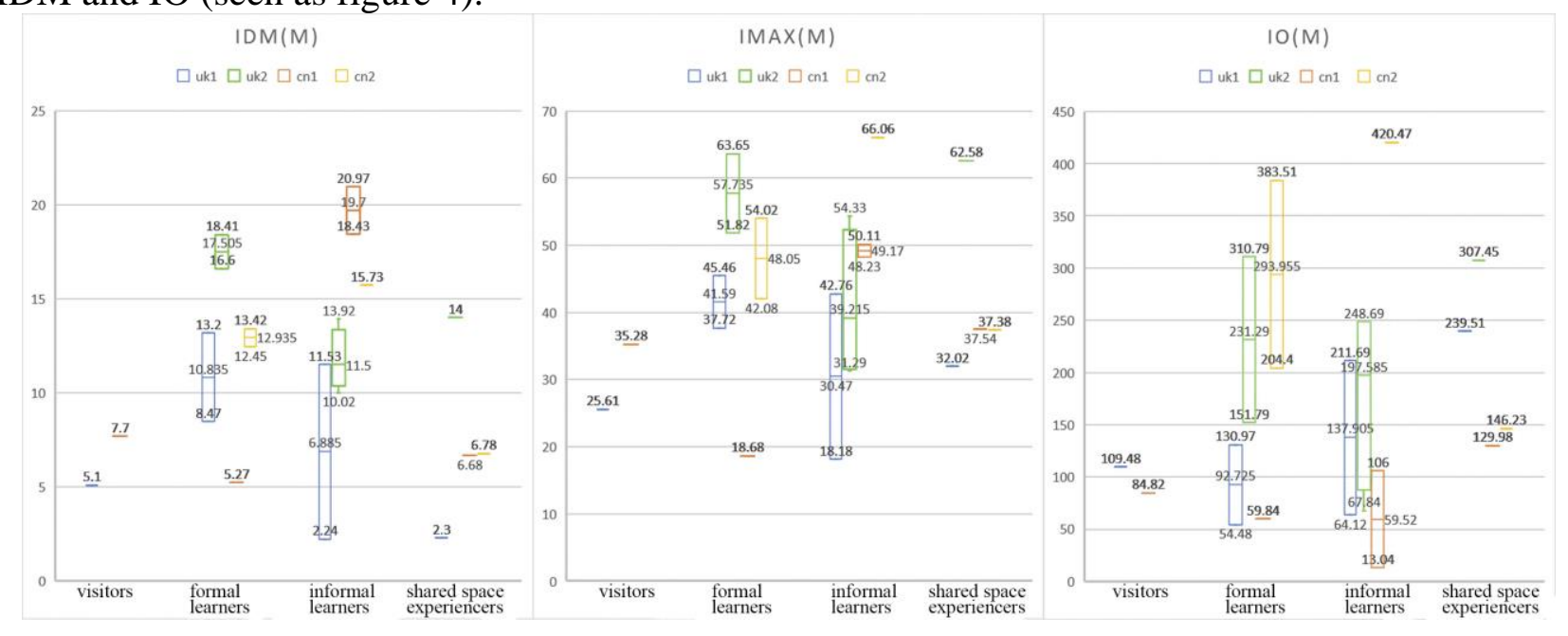

Fig. 4. Comparative analysis on box types in visual areas of $\mathrm{CN}$ and $\mathrm{UK}$ cases.

For the formal learners' perspective, the IDM value of UK2 is obviously higher than CN2, and the interval is similar. It means that the visual pull of the case standard layer in UK is larger than cases in China, while students and teachers are more easily to be guided by spaces.

For informal space learners, the IA mean value of CN2 (720.52) is higher than UK2 (551.18), and the IDM mean (66.06) is significantly higher than UK2 (39.215). CN2 has more visible space and a larger maximum viewing distance, which means greater exposure. The closure of CN2 (420.47) is also significantly greater than UK2 (197.585), indicating CN2 had a high degree of mystery, or provided an opportunity to hide or get lost. The spatial experience is more distinctive. 
Six types of learning spaces are linked with visual area analysis, while in this discussion, formal learners are selected to launch analysis on spatial types of learning areas covered by visual areas.

For UK cases, UK1 and UK2 clearly separated ordinary and professional classrooms into separate floors. In favor of teaching management, general education in UK2 is closer to informal learning space (mainly clustered and shared areas) than specialized education in UK1, after considering the needs of most users. The informal learning space in UK1 is for all users of the building, while UK2 maximizes the opportunities for students to use informal learning space before and after class.

In Chinese cases, as an office hall, $\mathrm{CN} 1$ also scattered gathering and sharing area in addition to general education. But in the formal learner's perspective, it is difficult to see any clustered or shared area easily. These areas are not prepared for users of general education leaners. There is no general education area in $\mathrm{CN} 2$, and there are a large number of specialized education areas. The gathering space is relatively remote, far from most of specialized education area and hard to be observed by majority of formal learners (the doors of classrooms).

For Chinese cases, learning space is relatively small and single in types, while UK cases are more abundant. Some classrooms in CN2 (on the left side of the plane) can't see the shared area or the clustering area, while formal learners in UK2 can see at least one shared area or the clustering area in front of the classroom door. Meanwhile, UK2 shared space has more privacy (less connected space around) with stronger accessibility. The outdoor reading space in UK2 has played a positive role in expanding the scope of informal learning activities, enhancing the spatial experience and promoting the generation of learning behavior.

\section{Discussion}

According to actual experience and photos, UK2-2 is better in UK cases comparing with UK2-1, while the actual visibility feature of standard floors in CN cases is between CN2-1 and CN2-2, closer to CN2-2. The second hypothetical studies proposed in this paper are reasonable.

According to visibility, factors affecting mean values of CON, VDM and VCC are not special area proportion. The relationship between local spaces and the layout of spatial morphology is important. The processing methods of bracings and areas of corridor interface are important reasons that affect the proportion of the high value in connectivity distribution and the low value in visual average depth distribution. The spaces with high connectivity, low VDM and low VCC spaces have good visual guidance and visibility.

According to visual area analysis, types of spaces for learning areas covered by formal learners represent visual experience for main users to the spaces around classrooms after classes. In UK cases, visual area of one visual point could cover one or even more shared and gathering spaces; for $\mathrm{CN}$ cases, seldom could cover two, while most of them only cover one or even none, which greatly affect users' judgment of surrounding learning environment. UK cases are more conducive to the promotion of informal learning activities.

\section{Conclusion}

In this study, we propose a processing mode for bracing spaces in a visibility analysis, which is more suitable for indoor atrium. Both visibility and inaccessibility of bracing spaces could be concerned. There are some particularities of outdoor courtyards which need to be further considered. Furthermore, a new idea of architectural analysis in colleges is put forward: operation mechanism and distribution rule of learning spaces are studied from the visibility and visual area features of spatial structures. In the design of learning environment, following points should be focused: 1 . The layout of public streamlines space form and the visibility relationship between local spaces; 2 . The coupling between the distribution of learning space and spatial visibility characteristics; 3 . Synergistic effect of different learning spaces.

In this study, samples are insufficient. Thus, further statistic demonstration is needed. Meanwhile, cases have no distinctions. Under different teaching concepts and methods, various types of learning 
buildings and environment at campuses naturally emerge. Such development is caused by current building and actual social needs. In future education architectures, cognition to special structure should be adapted to learning theories and methods in this digital era.

\section{Acknowledgement}

This research was financially supported by the National Undergraduate Training Program for Innovation and Entrepreneurship (Grant NO. 201713021010).

\section{References}

[1] Hillier, B., Space is the Machine: a Configurational Theory of Architecture,trans. By Yang Tao,Beijing:China Architecture and Beijing Press,2008,preface.

[2] Carranza, P.M., Koch, D., Izaki, A., 2013. SPOT with paths, and interactive diagram with a low complexity isovist algorithm. In:Kim, Y.O., Park, H.T., Seo, K.W. (Eds.), Proceedings of the Ninth International Space Syntax Symposium.. Sejong University, Seoul, pp. 1-13.

[3] Lu, Y., 2010. Measuring the structure of visual fields in nursing units. Health Environ. Res. Des. J. 3, 48-59.

[4] Morgareidge, D., Cai, H., Jia, J., 2014. Performance-driven design with the support of digital tools: applying discrete event simulation and space syntax on the design of the emergency department. Front. Archit. Res. 3, 250-264.

[5] Setola, N., Borgianni, S., Martinez, M., Tobari, E., 2013. The role of spatial layout of hospital public spaces in informal patient-medical staff interface. In: Kim, Y.O., Park, H.T., Seo, K.W. (Eds.), Proceedings of the Ninth International Space Syntax Symposium. Sejong University, Seoul, pp. 1-11.

[6] Cai, H., Zimring, C., 2013. Understanding cultural differences in nursing unit design with the support of space syntax analysis: are Chinese nursing units designs different from their U.S. counterparts?. In: Kim, Y.O., Park, H.T., Seo, K.W. (Eds.), Proceedings of the Ninth International Space Syntax Symposium. Sejong University, Seoul, pp. 1-24.

[7] Stamps, A.E., 2005. Isovists, enclosure, and permeability theory. Environ. Plan. B: Plan. Des. 32, 735-762.

[8] Dawes, M.J., Ostwald, M.J., 2014. Prospect-Refuge theory and the textile-block houses of Frank Lloyd Wright: an analysis of spatiovisual characteristics using isovists. Build. Environ. 80, 228-240.

[9] Dosen, A.S., Ostwald, M.J., 2017. Lived space and geometric space: comparing people's perceptions of spatial enclosure and exposure with metric room properties and isovist measures. Archit. Sci. Rev. 60, 62-77.

[10]Turner, A. and Penn, A. (2002) Encoding Natural Movement as an Agent-based System: an Investigation into Human Pedestrian Behaviour in the Built Environment. Environment and Planning B, 29(4), 473-490. pp.483-484. 